The very short proof of 4.1 depends on the nontrivial result of Hewitt and Zuckerman [3, Theorem 8.10] that a proper $H$-semigroup always has a semicharacter $f$ such that $0<f<1$. The use of this fact is avoided in the following argument, suggested by the referee: let $G$ be a linear semigroup, $H$ its group of quotients, and $G^{\prime}$ the set of $x$ in $H$ such that $x^{n} \in G$ for some $n \in N$. In addition to being positively ordered and archimedean, $G^{\prime}$ is also naturally ordered and hence, by a theorem of Hölder and Huntington (see [1]), $G^{\prime}$ is isomorphic to a subsemigroup of $R^{+}$; so is $G$.

\title{
REFERENCES
}

1. A. H. Clifford, Totally ordered commutative semigroups, Bull. Amer. Math. Soc. 64 (1958), 305-316.

2. E. Hewitt and H. S. Zuckerman, Finite dimensional convolution algebras, Acta Math. 93 (1955), 67-119.

3. - The $h_{1}$-algebra of a commutative semigroup, Trans. Amer. Math. Soc. 83 (1956), 70-97.

4. E. Hille and R. S. Phillips, Functional analysis and semigroups, Amer. Math. Soc. Colloq. Publ. Vol. 31, Amer. Math. Soc., Providence, R. I., 1957.

5. K. A. Ross, $A$ note on extending semicharacters of semigroups, Proc. Amer. Math. Soc. 10 (1959), 597-583.

UnIVERSITY OF CoLORADO

\section{REPRESENTATIVE SETS AND DIRECT SUMS}

\section{ROBERT RYAN ${ }^{1}$}

1. Let $(X, \Lambda, \mu)$ be a complete measure space and denote by $N$ the collection of all locally $\mu$-null subsets of $X$, i.e., $E \in N$ if and only if $\mu(E \cap F)=0$ for all $\mu$-summable sets $F$. For $E$ and $F$ in $\Lambda$ we write $E \equiv F$ if and only if $E \Delta F \in N$ where $E \Delta F=(E-F) \cup(F-E)$. The relation $E \equiv F$ is clearly an equivalence relation.

$J$. von Neumann showed that if $\mu$ is $\sigma$-finite there exists a mapping $\rho$ of $\Lambda$ into $\Lambda$ with the following properties:

(1) $\rho(E) \equiv E$;

(2) $E \equiv F$ implies $\rho(E)=\rho(F)$;

(3) $\rho(\varnothing)=\varnothing, \rho(X)=X$ ( $\varnothing=$ the empty set);

(4) $\rho(E \cap F)=\rho(E) \cap \rho(F)$;

(5) $\rho(E \cup F)=\rho(E) \cup \rho(F)$.

Received by the editors January 10, 1963 and, in revised form, February 18, 1963.

1 Sponsored by the Mathematics Research Center, U. S. Army, Madison, Wis consin, under Contract No. DA-11-022-ORD-2059. 
However, von Neumann's published proof in [3] applies only when $X=R^{n}$ and $\mu$ is the Lebesgue measure. An independent proof for a general $\sigma$-finite measure space was later given by D. Maharam [2]. By a still different proof, using Banach algebra methods, A. and C. Ionescu Tulcea showed in [1] that the result also holds when $X$ is locally compact and $\mu$ is a positive Radon measure.

When this result holds we will say that $(X, \Lambda, \mu)$ has property $(M)$. The purpose of this note is to show that property $(\mathrm{M})$ is equivalent to $X$ being the direct sum of $\mu$-summable subsets $\left\{Y_{\alpha}: \alpha \in A\right\}$ plus possibly a locally $\mu$-null set. $\$ 2$ contains some preliminary remarks on the contraction of $\mu$ and direct sums. $\$ 3$ contains the main result. For further results about the material in $\$ 2$ we refer to the paper by A. C. Zaanen [5]. In this connection we also mention I. E. Segal's fundamental paper on localizable spaces [4]. The author wishes to thank the referee for several useful suggestions incorporated in this paper.

2. The contraction of $\mu$ is defined on $\Lambda$ by $\mu_{c}(E)=\sup \mu(F)$ over all $F \subset E$ with $\mu(F)<\infty$. It can easily be shown that $\mu_{c}$ is a measure on $\Lambda$ and that the $\mu_{c}$-measurable subsets of $X$ are just the $\mu$-measurable subsets $\Lambda$, i.e., the Carathéodory extension of $\mu_{c}$ yields no new $\mu_{c}$-measurable sets. A set $E \in \Lambda$ is $\mu_{c}$-null if and only if $E$ is locally $\mu$-null. Thus, the space $\left(X, \Lambda, \mu_{c}\right)$ is complete if and only if $(X, \Lambda, \mu)$ is complete. This proves the following:

Lemma 1. ( $X, \Lambda, \mu)$ has property (M) if and only if $\left(X, \Lambda, \mu_{c}\right)$ has property (M).

We will say that $X$ is a direct sum of $\mu$-summable sets $\left\{Y_{\alpha}: \alpha \in A\right\}$ whenever $X-Y=\mathrm{U}_{\alpha \in A} Y_{\alpha}$, where $Y$ is either a locally $\mu$-null set with $\mu(Y)=\infty$ or $Y=\varnothing$, where the sets $\left\{Y_{\alpha}\right\}$ are mutually disjoint $\mu$ summable sets with positive $\mu$-measure and where every $\mu$-summable set $E$ is the union, except for a $\mu$-null set, of at most a countable number of the sets $\left\{E \cap Y_{\alpha}\right\}$.

We will assume that $\mu(X) \neq 0$ and that $X$ contains some $\mu$-summable set with positive measure. Thus, if $X$ is a direct sum then $A$ is not empty.

LeMma 2. $X$ is a direct sum of $\mu$-summable sets if and only if $X$ is a direct sum of $\mu_{c}$-summable sets.

Proof. Suppose that $X$ is a direct sum of $\mu$-summable sets $\left\{Y_{\alpha}: \alpha \in A\right\}$. Then $Y=X-\bigcup_{\alpha \in A} Y_{\alpha}$ is a $\mu_{c}$-null set. By adding $Y$, which may be empty, to some set $Y_{\alpha_{0}}$ we have that $X=\left(\bigcup_{\alpha \neq \alpha_{0}} Y_{\alpha}\right)$ $\cup\left(Y_{\alpha_{0}} \cup Y\right)$ is a direct sum of $\mu_{c}$-summable sets. 
Now, assume that $X$ is a direct sum $X=\bigcup_{\alpha \in A} X_{\alpha}$ of $\mu_{c}$-summable sets $\left\{X_{\alpha}: \alpha \in A\right\}$. (The set $Y$ does not appear since locally $\mu_{c}$-null sets are $\mu_{c}$-null.) By Lemma 10.1 of [5] there exists for each $\alpha \in A$ a $\mu$-summable set $Y_{\alpha} \subset X_{\alpha}$ such that $\mu\left(Y_{\alpha}\right)=\mu_{c}\left(X_{\alpha}\right)$. Thus $X_{\alpha}-Y_{\alpha}$ is locally $\mu$-null. If $X_{\alpha}$ happens to be $\mu$-summable we choose $Y_{\alpha}=X_{\alpha}$ otherwise we have $\mu\left(X_{\alpha}-Y_{\alpha}\right)=\infty$. Theorem 8.6 of [5] implies that $Y=\mathrm{U}_{\alpha \in A}\left(X_{\alpha}-Y_{\alpha}\right)$ is measurable and $\mu_{c}$-null. Hence $Y$ is locally $\mu$-null and $\mu(Y)=\infty$ or $Y=\varnothing$.

Let $E$ be any $\mu$-summable subset of $X$. Then $E$ is $\mu_{c}$-summable and $\mu(E)=\mu_{c}(E)$. By hypothesis there exists a countable number of sets $\alpha=\alpha_{1}, \alpha_{2}, \cdots$ such that $\mu_{c}\left(E-\bigcup_{i=1}^{\infty}\left(E \cap X_{\alpha_{i}}\right)\right)=0$. Since $\mu\left(E \cap Y_{\alpha}\right)$ $=\mu_{c}\left(E \cap X_{\alpha}\right)$ we have

$$
\mu\left(E-\bigcup_{i=1}^{\infty}\left(E \cap Y_{\alpha_{i}}\right)\right)=\mu_{c}\left(E-\bigcup_{i=1}^{\infty}\left(E \cap X_{\alpha_{i}}\right)\right)=0 .
$$

This proves that $X$ is a direct sum of $\mu$-summable sets $\left\{Y_{\alpha}: \alpha \in A\right\}$.

3. We can now state and prove the main result:

Theorem. $(X, \Lambda, \mu)$ has property (M) if and only if $X$ is a direct sum of $\mu$-summable sets $\left\{Y_{\alpha}: \alpha \in A\right\}$.

Proof. From Lemmas 1 and 2 we can see that the statement of the theorem is equivalent to the following statement: $\left(X, \Lambda, \mu_{c}\right)$ has property (M) if and only if $X$ is a direct sum of the $\mu_{c}$-summable sets $\left\{X_{\alpha}: \alpha \in A\right\}$. This is the statement we will prove.

Suppose that $X=\bigcup_{\alpha \in A} X_{\alpha}$ is a direct sum of $\mu_{c}$-summable sets. By the result of $\mathrm{D}$. Maharam [2] there exists for each $\alpha \in A$ a mapping $\rho_{\alpha}$ of $\Lambda \cap X_{\alpha}$ into $\Lambda \cap X_{\alpha}$ satisfying (1)-(5). Using in general the axiom of choice, choose such a $\rho_{\alpha}$ for each $\alpha \in A$. Define $\rho$ on $\Lambda$ by $\rho(E)=\bigcup_{\alpha \in A} \rho_{\alpha}\left(E \cap X_{\alpha}\right)$. It is a consequence of Theorem 8.6 of [4] that $\rho(E)$ is measurable and that $\mu_{c}[\rho(E) \Delta E]=0$. Thus $\rho$ satisfies (1); $\rho$ clearly satisfies (2)-(5).

To prove the other half of the theorem we assume that $\left(X, \Lambda, \mu_{c}\right)$ has the property (M). Let $\rho$ be one of the mappings of $\Lambda$ into $\Lambda$ satisfying (1)-(5). Let $E^{*}$ denote the class of all sets $F \in \Lambda$ such that $\mu_{c}(E \Delta F)=0$. Following Zaanen [5, p. 178] we consider all collections $\mathcal{F}=\left\{X_{\alpha}^{*}\right\}$ corresponding to sets $X_{\alpha} \in \Lambda$ for which $0<\mu_{c}\left(X_{\alpha}\right)<\infty$ and $\mu_{c}\left(X_{\alpha} \cap X_{\alpha^{\prime}}\right)=0$ for $\alpha \neq \alpha^{\prime}$. The assumption that $X$ contains some $\mu$ summable set with positive measure implies that the family $\{F\}$ is not empty. The family $\{\mathcal{F}\}$ is partially ordered by inclusion and it is clear that every chain has a least upper bound. Thus, by Zorn's lemma there exists a maximal element. Let $F_{m}=\left\{X_{\alpha}^{*}: \alpha \in A\right\}$ be a fixed maximal element. 
Consider the sets $\rho\left(X_{\alpha}\right)$. For each $\alpha \in A, 0<\mu_{c}\left[\rho\left(X_{\alpha}\right)\right]<\infty$ and $\rho\left(X_{\alpha}\right) \cap \rho\left(X_{\alpha^{\prime}}\right)=\rho\left(X_{\alpha} \cap X_{\alpha^{\prime}}\right)=\varnothing$ for $\alpha \neq \alpha^{\prime}$ by (3). If $E$ is an arbitrary $\mu_{c}$-summable set then by Theorem 8.4 of $[4], \mu_{c}\left[E \cap \rho\left(X_{\alpha}\right)\right]>0$ for an at most countable number of indices $\alpha=\alpha_{1}, \alpha_{2}, \cdots$ and $\mu_{c}\left[E-\bigcup_{i=1}^{\infty}\left(E \cap \rho\left(X_{\alpha_{i}}\right)\right)\right]=0$. This shows that

$$
E \cap\left[X-\bigcup_{\alpha \in A} \rho\left(X_{\alpha}\right)\right]=E-\bigcup_{\alpha \in A}\left[E \cap \rho\left(X_{\alpha}\right)\right]
$$

is a subset of a $\mu_{c}$-null set for each $\mu_{c}$-summable set $E$. Since $\left(X, \Lambda, \mu_{c}\right)$ is assumed to be complete, this proves that $X-\bigcup_{\alpha \in A} \rho\left(X_{\alpha}\right)$ is a $\mu_{c}$-null set and hence measurable. Now write $Z_{\alpha_{0}}=\rho\left(X_{\alpha_{0}}\right) \cup\left[X-U_{\alpha \in A} \rho\left(X_{\alpha}\right)\right]$ and $Z_{\alpha}=\rho\left(X_{\alpha}\right)$ for $\alpha \neq \alpha_{0}$. Then it is clear that $X=\bigcup_{\alpha \in A} Z_{\alpha}$ is a direct sum of $\mu_{c}$-summable sets $\left\{Z_{\alpha}: \alpha \in A\right\}$ ( $\alpha_{0}$ is an arbitrary element of the index set $A$ ). This proves the theorem.

This theorem combined with Theorems 9.2 and 10.2 of [5], gives the following results:

Corollary. If $(X, \Lambda, \mu)$ has the property (M) then the measure $\mu_{c}$ is localizable or, equivalently, $\mu$ has the Radon-Nikodym property.

\section{REFERENCES}

1. A. and C. Ionescu Tulcea, On the lifting property, J. Math. Anal. Appl. 3 (1961), 537-546.

2. D. Maharam, On a theorem of von Neumann, Proc. Amer. Math. Soc. 9 (1958), 987-994.

3. J. von Neumann, Algebraische Repräsentanten der Funktionen bis auf eine Menge von Masse Null, J. Crelle 165 (1931), 109-115.

4. I. E. Segal, Equivalences of measure spaces, Amer. J. Math. 73 (1951), 275-313.

5. A. C. Zaanen, The Radon-Nikodym theorem. II, Indag. Math. 23 (1961), 171187.

UNIVERSITY OF WISCONSIN 